\title{
La Escama Lobada de Laca, Paratachardina lobata (Chamberlin) (Insecta: Hemiptera: Sternorrhyncha: Coccoidea: Kerriidae) ${ }^{1}$
}

\author{
F. W. Howard, Robert Pemberton, Avas Hamon, Greg S. Hodges, Catharine M. Mannion, David \\ McLean Jeanette Wofford ${ }^{2}$
}

\section{Introducción}

Una especie de insecto de escama que es nueva en la Florida es potencialmente una de las plagas más devastadoras de arboles y arbustos en la historia del estado. La escama lobada de laca, Paratachardina lobata (Chamberlin)(Hemiptera: Sternorrhyncha: Coccoidea: Kerriidae), un insecto de escama nativo de la India y Sri Lanka, se encontró por primera vez en la Florida en agosto de 1999 por personal del Departamento de Agricultura y Servicios para Consumidores, División de Industria de Plantas (DIP)(Hamon 2001). La identificación de la especie por Avas Hamon de DIP fue confirmada por D. R. Miller del laboratorio de Entomología Sistemática, Departamento de Agricultura de los Estados Unidos, Beltsville, Maryland. Este registro fue sobre hibisco (Hibiscus rosa-sinensis) en el pueblo de Davie (Condado de Broward). La planta fue destruida por el personal del DIP. Las plantas en la vecindad a esta planta infestada fueron inspeccionadas sin encontrar $P$. lobata. Sin embargo, la especie fue encontrada otra vez en el 2000 sobre un caucho (Ficus benjamina) en Davie, sobre icaco (Chrysobalanus icaco) en Weston (Condado de Broward), y sobre icaco en dos sitios en Miami (Condado de Miami- Dade). En el 2001, la especie de insecto de escama se encontró en 11 sitios en el condado de Broward y en seis sitios en el Condado de Miami-Dade. En diciembre del 2001, los inspectores del DIP encontraron P. lobata en Lake Worth (Condado de Palm Beach). A partir de octubre del 2002, P. lobata se ha registrado en sitios desde Lake Worth al norte hasta Homestead (Condado de Miami-Dade) en el sur, esto es, una distancia de 128 $\mathrm{km}$, y desde la costa hasta $28 \mathrm{~km}$ al interior. Después de encontrarse este insecto de escama en la Florida, unos ejemplares de la misma especie fueron encontrados en la Colección Estatal de la Florida de Artrópodos los cuales fueron colectados en 1992.

1. This document is EENY-309, one of a series of Featured Creatures from the Entomology and Nematology Department, Florida Cooperative Extension Service, Institute of Food and Agricultural Sciences, University of Florida. Publicación: Septiembre 2003. Revised: April 2004, May 2004. Derechos reservados 2003-2004. This document is also available on Featured Creatures Website at http://creatures.ifas.ufl.edu. Please visit the EDIS Website at http://edis.ifas.ufl.edu. Additional information on these organisms, including many color photographs, is available at the Entomology and Nematology Department website at http://entnemdept.ifas.ufl.edu/.

2. F. W. Howard, University of Florida; Robert Pemberton, USDA; Avas Hamon, Division of Plant Industry; Greg S. Hodges, Division of Plant Industry; Catharine M. Mannion, University of Florida; David McLean, Broward Community College and Nova Southeastern University; and Jeanette Wofford, Cooper City Arborist, Traductor (Inglés a Español). F. W. Howard Versión en español revisada por Juan Sebastián Ortiz, Loreto Castro (University of Florida), y Rosalinda Leidi (USDA, ARS).

The Institute of Food and Agricultural Sciences (IFAS) is an Equal Employment Opportunity - Affirmative Action Employer authorized to provide research, educational information and other services only to individuals and institutions that function without regard to race, creed, color, religion, age, disability, sex, sexual orientation, marital status, national origin, political opinions or affiliations. For information on obtaining other extension publications, contact your county Cooperative Extension Service office. Florida Cooperative Extension Service / Institute of Food and Agricultural Sciences / University of Florida / Larry R. Arrington, Interim Dean 


\section{Descripción y biología}

Las hembras adultas de P. lobata, con la escama miden alrededor de 1.5-2.0 mm de largo y casi lo mismo de ancho. La escama tiene dos pares de lóbulos prominentes. $\mathrm{Al}$ ojo experto, la forma de ' $\mathrm{X}$ ' de este insecto de escama es apreciable, hasta sin magnificación. La escama es extremamente dura y quebradiza, lustrosa y de un color granate-castaño, pero frecuentemente está cubierta por una capa de fumagina por lo cual aparece ser de un color negro-opaco. La hembra de la escama lobada de laca, como es el caso con cóccidos en general, se desarrolla durante dos instares, y muda al tercer estadio, lo cual es la hembra adulta. Los primeros instares son ovalados-alargados y alrededor de 0.4 $\mathrm{mm}$ de largo. La forma lobata característica se desarrolla en el segundo instar, y es más pronunciada en la adulta. No se han observado los machos de esta especie en la Florida.

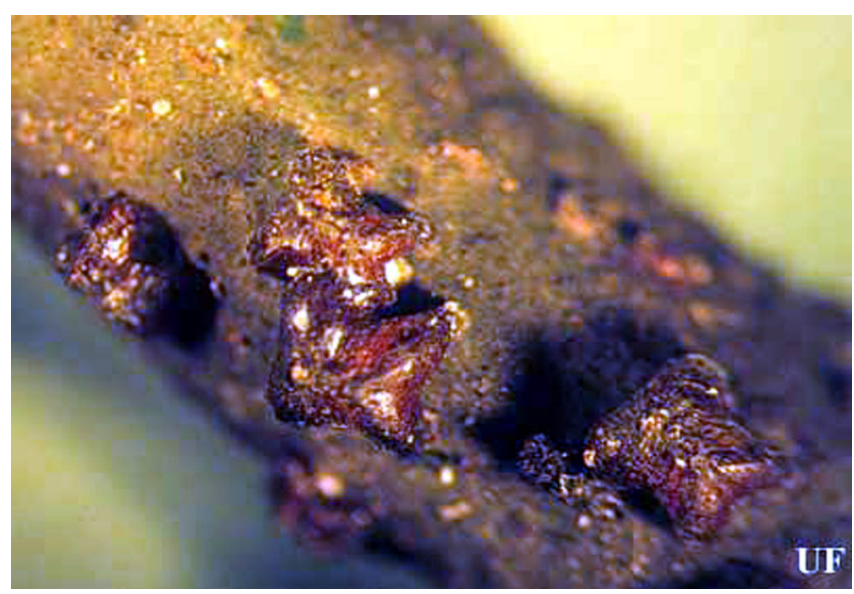

Figure 1. Hembras maduras de la escama lobada de laca, Paratachardina lobata (Chamberlin). Credits: Fotografa: F. W. Howard, University of Florida

Como las hembras adultas de los insectos de escama no tienen alas, no juegan ningún papel en la dispersión de poblaciones para ocupar nuevas plantas hospedantes. Los insectos de escamas dependen en dispersión pasiva del primer instar vía corrientes de aire. Foresis (transporte por pájaros y otros animales) puede ser de alguna importancia en algunas especies. Sin duda, el movimiento de plantas hospedantes infestadas entre áreas urbanas es un factor clave para la dispersíon los insectos de escama.

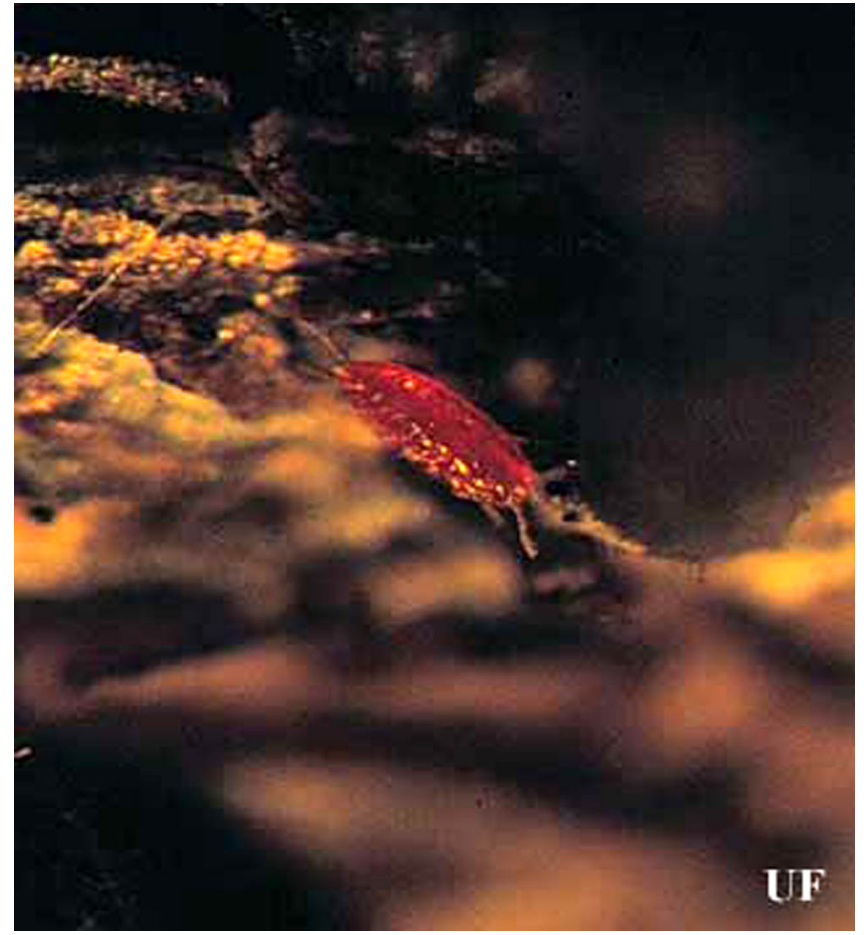

Figure 2. Primer instar de la escama lobada de laca, Paratachardina lobata (Chamberlin). Credits: Fotografa: F. W. Howard, University of Florida

\section{La familia de los insectos de laca}

La escama lobada de laca pertenece a la familia de cóccidos de laca, Kerriidae, la especie mejor conocida de la cual es el verdadero cóccido de laca, Kerria lacca (Kerr). La escama de este se ha utilizado para hacer goma laca y productos similares. Pero la mayoría de especies de esta familia, incluyendo $P$. lobata, no producen ningún material de valor comercial. El nombre científico especifico, lobata, se refiere a las cuatro proyecciones prominentes, $o$ lóbulos, de esta escama, y el nombre vernáculo, 'escama lobada de laca' puede ser aplicado a esta especie.

De las 28 familias de insectos de escama (súperfamilia Coccoidea) reconocidas por Miller y Ben-Dov (2002), 11 son representadas por especies las cuales son nativas a la Florida (Aclerdidae, Asterolecaniidae, Diaspididae, Cerococcidae, Coccidae, Conchaspidae, Eriococcidae, Kermesidae, Margarodidae, Ortheziidae, y Pseudococcidae.) No hay ninguna especie de Kerriidae nativa de la Florida y tierras adyacentes. La familia Kerriidae está limitada sobre todo a las latitudes tropicales, con una minoría de especies presentes en áreas desérticas 
de baja latitud. De las 87 especies descritas, 64 se encuentran en el Hemisferio Oriental. De las especies nativas del Hemisferio Occidental, 13 son registradas para América del Sur, seis para México (dos de las cuales son registradas para el suroeste de los Estados Unidos), tres registradas solamente en el suroeste de los EE.UU. y una especie en Jamaica (Ben-Dov 2002).

\section{Los efectos sobre las plantas hospedantes}

Paratachardina lobata se ha encontrado sobre todo infestando plantas leñosas dicotiledóneas. Infesta las ramitas y ramas pequeñas y los troncos de menos de $2 \mathrm{~cm}$ de diámetro. No se ha encontrado sobre follaje.

Sobre las plantas hospedantes altamente susceptibles, los insectos de escamas estan abarrotados, formando una masa contigua la cuál aparece como una costra oscura llena de bultos. Se han contado hasta 19 hembras maduras por $100 \mathrm{~mm} 2$ en ramitas de palo de cera (Myrica cerifera), una planta hospedante altamente susceptible.

Infestaciones densas se asocian con la muerte de ramas de algunas especies de plantas; en casos severos, arbustos y árboles pequeños altamente infestados se han muerto. El palo de cera está especialmente propenso a tener infestaciones densas y morir por los efectos de la escama lobata. Algunas especies de plantas parecen tolerar las infestaciones densas, pero eso puede ser ilusorio, pues todavía no se saben los efectos de tales infestaciones a largo plazo.

\section{Rango de plantas hospedantes de la escama}

Este insecto de escama se ha encontrado solamente sobre plantas dicotiledóneas, sobre una especie conífera (enebro rojo del sur, Juniperus silicicola) y sobre una palma (palma datilera miniatura, Phoenix roebelenii). A partir de octubre del 2002, más de 120 especies en 44 familias de plantas leñosas han sido identificadas como plantas hospedantes en la Florida (Cuadros $1 \& 2$ ). Estas incluyen 39 especies nativas a la Florida. La mayoría de las plantas hospedantes exóticas se

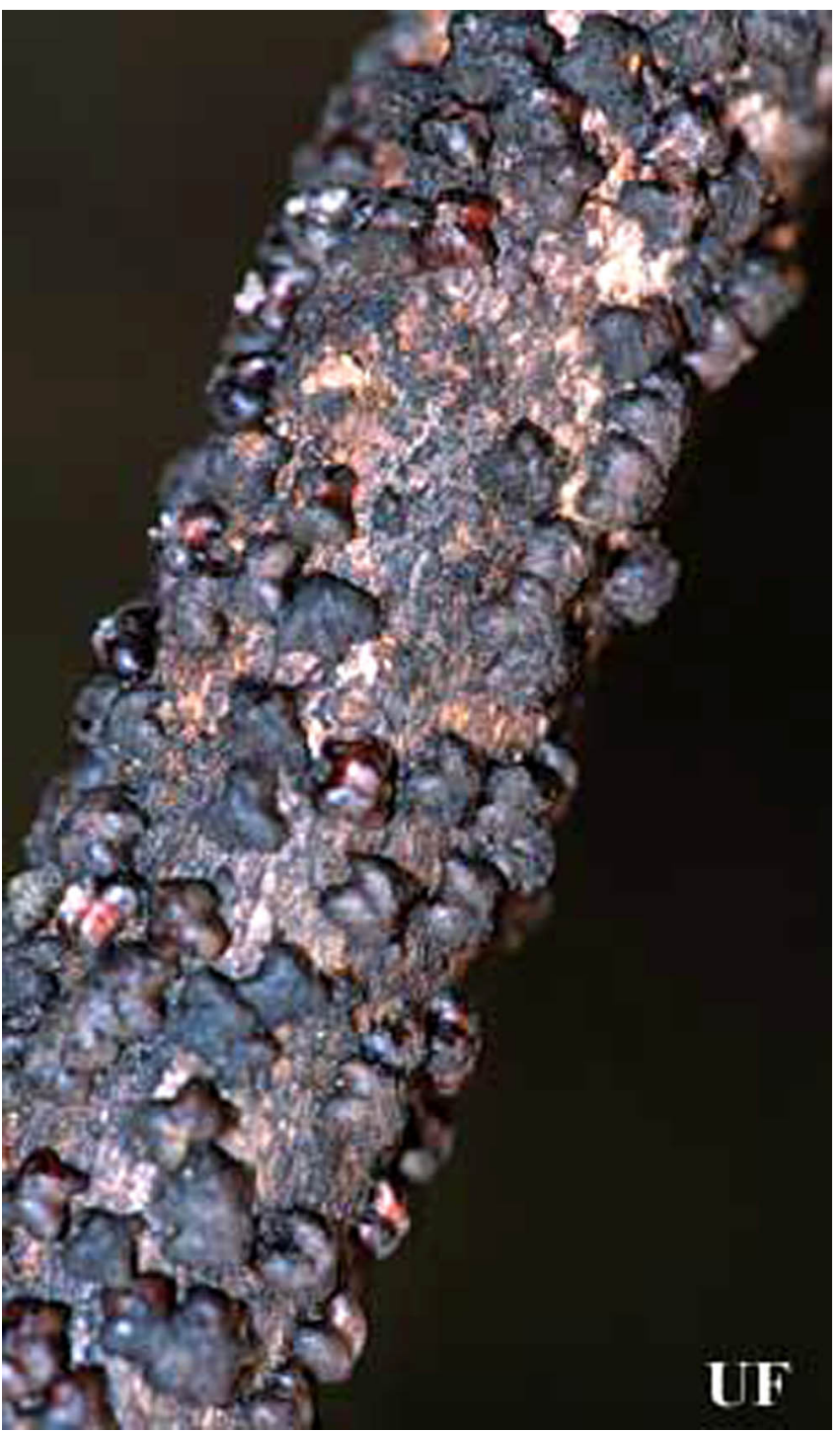

Figure 3. Ramita de palo de cera infestada de la escama lobada de laca, Paratachardina lobata (Chamberlin). Credits: Fotografa: F. W. Howard, University of Florida

cultivan como árboles o arbustos ornamentales, o como árboles frutales. Algunas de estas especies son sumamente importantes en los paisajes urbanos como árboles de sombra, árboles ejemplares, o cercas vivas. Algunas familias de plantas, notablemente Fabaceae, Myrtaceae, y Moraceae, son bien representadas por especies que sirven como plantas hospedantes, pero eso puede estar relacionado con su abundancia en el paisaje o debido a otro prejuicio. Las plantas en diferentes sitios han sido expuestas a las infestaciones por diferentes períodos, y por eso, los niveles de infestación son variables. Diferencias en susceptibilidad no han sido determinadas en experimentos. Sin embargo, ciertas especies parecen ser altamente susceptibles, 


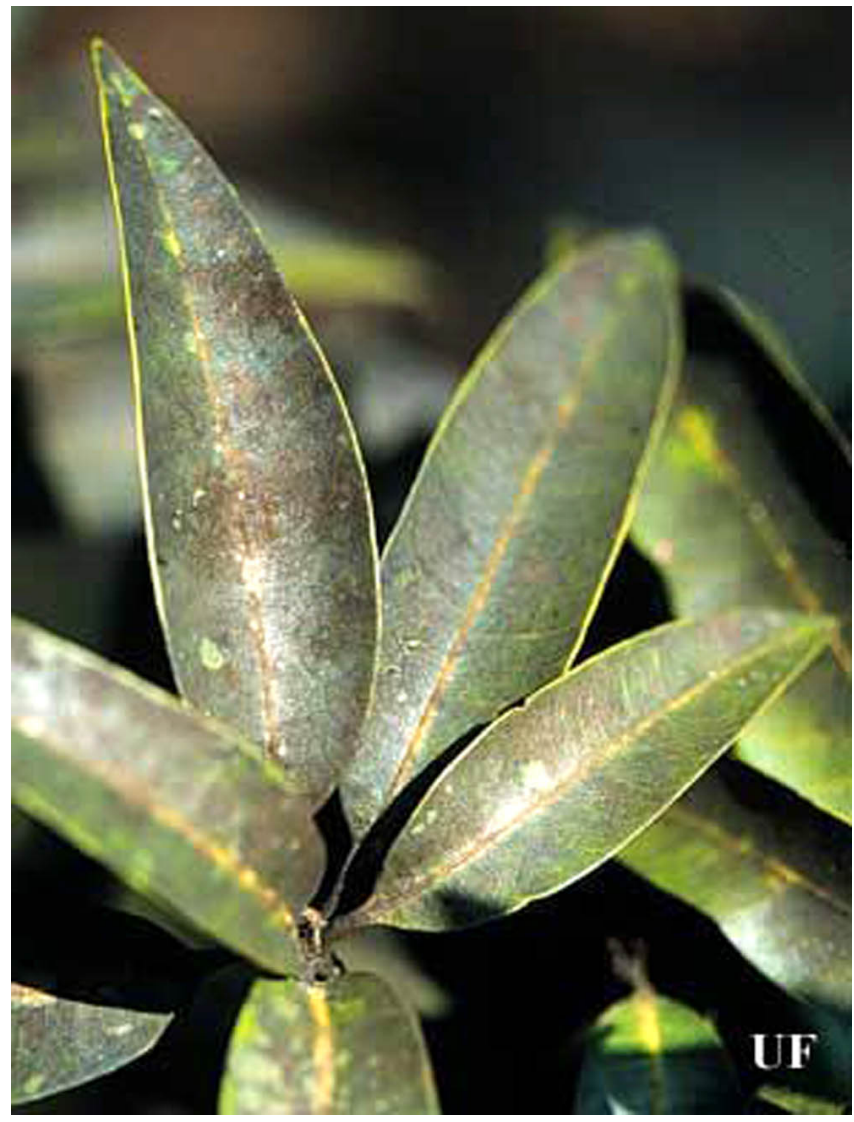

Figure 4. Fumagina sobre hojas de mango, resultado indirecto de una infestación de la escama lobada de laca, Paratachardina lobata (Chamberlin). Credits: Fotografa: F. W. Howard, University of Florida
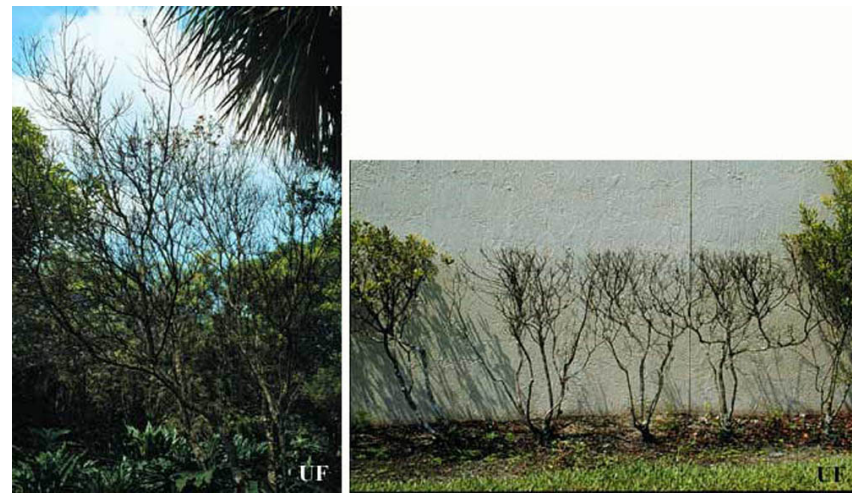

Figure 5. Palo de cera en bosque natural (izquierda) y como cerca viva (derecho), muertos por causa de infestaciones de la escama lobada de laca, Paratachardina lobata (Chamberlin). Credits: Fotografa: F. W. Howard, University of Florida

incluyendo algunas especies nativas, e. g., palo de cera, icaco (Chrysobalanus icaco), mangle botón (Conocarpus erectus), higüero estrangulador (Ficus aurea), myrsine (Myrsine guianensis), laurel rojo (Persea borbonia), y café-silvestre (Psychotria nervosa); plantas ornamentales exóticas, e. g., ucar (Bucida buceras), laurel de la India (Ficus microcarpa
), caucho ( $F$. benjamina); y árboles frutales, e. g., lychee (Litchi chinensis), mango (Mangifera indica), y carambola (Averrhoa carambola).

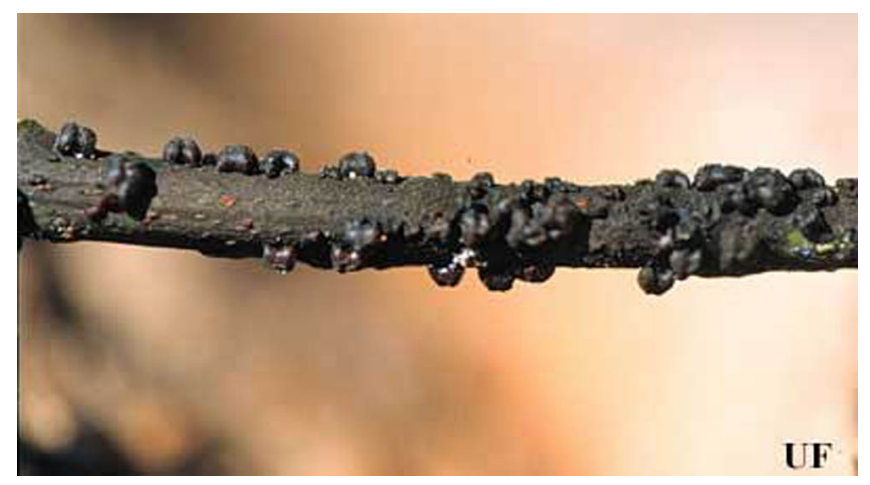

Figure 6. Ramita de mango infestada de la escama lobada de laca, Paratachardina lobata (Chamberlin). Credits: Fotografa: F. W. Howard, University of Florida

\section{Cuadro 1. Lista de plantas hospedantes de escama lobada de laca hasta Octubre, 2002}

Lista compilada por Bill Howard, Dave McLean, Bob Pemberton, Avas Hamon, Greg Hodges, y Jeanette Wofford. Contribuidores adicionales: John Cannon, Chris y Karolynne Griffiths, Way Hoyt, Neal Kolnik, Andrew Southerland y Laura Tooley.

* indica especies nativas á la Florida.

Acacia auriculiformis Bentham (Fabaceae) - acacia de hoja-oreja

Acalypha wilkesiana Mueller (Eurphorbiaceae) acalifa

*Acer rumbrum L. (Aceraceae) - arce rojo

Annona cherimola Miller X A. squamosa L.

(Annonaceae) - atemoya

*Annona glabra L. (Annonaceae) - anón de agua

Annona muricata L. (Annonaceae)- guanábana

Annona reticulata L. (Annonaceae)- mamón

Annona squamosa L. (Annonaceae)- anón

Antidesma bunius (L.) Sprengel (Euphorbiaceae) bignay 
Ardisia escallanoides Schlechtendal \& Chamisso (Myrsinaceae) - baya de marga

Averrhoa carambola L. (Oxalidaceae) - carambola Avicennia germinans (L.) L. (Verbenaceae) mangle negro

*Baccharis halimifolia L. (Asteraceae )- carqueja

Bauhinia sp. (Fabaceae) - árbol de las orquídeas

Blighia sapida Konig (Sapindaceae) - acki

Brosimum alicastrum Swartz (Moraceae) - ramón, cacique

Brunfelsia sp. (Solanaceae)

Brunfelsia nitida Bentham (Solanaceae) - dama de noche

Brya ebenus (L.) de Candolle (Fabaceae) granadillo

Bucida buceras L.(Combretaceae) - ucar

Bumelia celastrina Humboldt, Bonpland \& Kunth (Sapotaceae) - coma

*Bursera simaruba (L.) Sargent (Burseraceae) almácigo

Calliandra haematocephala Hasskarl (Fabaceae) caliandra

Calliandra surinamensis (Fabaceae) - caliandra rosa

Callistemon viminalis (Gaertner) Cheel

(Myrtaceae) - calistemon

Calophyllum brasiliense Cambessédes

(Clusiaceae) - palo maría

*Calyptranthes pallens Grisebach (Myrtaceae) limoncillo

*Calyptranthes zuzygium (L.) Swartz (Myrtaceae)

- arrayán del rió

Cananga odorata (Lam ) J. D. Hooker \& T.

Thomson (Annonaceae) - ilan-ilan
*Capparis cynophallophora L. (Capparaceae) burro prieto

Casuarina equisetifolia L. (Casuarinaceae) casuarina

Celtis laevigata Willdenow (Celtidacae) - palo blanco

Cestrum nocturnum L. (Solanaceae) - galán de noche

*Chrysobalanus icaco L. (Chrysobalanaceae) icaco

Chrysophyllum cainito L. (Sapotaceae) - caimito

*Chrysophyllum oliviforme L. (Sapotaceae) caimitillo

Cinnamomum zeylanicum Blume (Lauraceae) canela

Citrus X paradisi Macfadyen (Rutaceae) - toronjo

Clausena lansium (Loureiro) Skeels (Rutaceae) wampi

*Clusia rosea Jacquin (Clusiaceae) - cupey

*Conocarpus erectus L. (Combretaceae) - mangle botón

Cupaniopsis anacardioides (A. Richard) Radlkofer (Sapindaceae) - carrotwood

Diospyros digyna Jacquin (Ebenaceae) - zapote negro

*Dipholis salicifolia (L.) Alphonse De Candolle (Sapotaceae) - zitzia

Dovyalis hebecarpa (G. Gardner) Warburg

(Flacourtiaceae) - grosella de Ceilán

Elaeocarpus sp. (Elaeocarpaceae)

*Eugenia axillaris (Swartz) Willdenow

(Myrtaceae) - guairaje

Eugenia brasiliensis Lamarck (Myrtaceae) grumichama 
Eugenia uniflora L. (Myrtaceae) - pitanga

Eugenia confusa De Candolle (Myrtaceae) arrayán de baya roja

* Exothea paniculata (Jussieu) Radlkofer (Sapindaceae)- guamacá, yaicuaje

*Ficus aurea Nuttall (Moraceae) - jagüey estrangulador

Ficus benjamina L. (Moraceae) - caucho

Ficus microcarpa L.f. (Moraceae) - laurel de la India

Ficus nota (Blanco) Merrill (Moraceae) - tibig

Ficus rubiginosa Ventenat (Moraceae) - jagüey herrumboso

Ficus salicifolia (Vahl) Berg (Moraceae) - jagüey de hoja de sauce

Filicium decipiens (Wight \& Arnot) Thwaites (Sapindaceae) - árbol-helecho japonés

Fortunella japonica (Thunberg) Swingle

(Rutaceae) - naranjito

Garcinia prainiana King (Clusiaceae) - mangostan botón

Gardenia jasminoides Ellis (Rubiaceae) - gardenia Grewia occidentalis L. (Tiliaceae) - flor-estrella

Hamelia cuprea Grisebach (Rubiaceae) - Bahamas flame bush

*Hamelia patens Jacquin (Rubiaceae) - coloradillo

Hibiscus rosa-sinensis L. (Malvaceae) - hibiscus

*Ilex vomitoria Aiton (Aquifoliaceae) - acebo yaupon

Inga affinis De Candolle (Fabaceae) - ingá dulce

Inga edulis Martius (Fabaceae) - guamo

santafereño, guaba, inga-cipo

*Juniperus silicicola (Small) Bailey (Cupressacae)

- enebro rojo del sur
*Krugiodendron ferreum (Vahl) Urban

(Rhamnaceae) - bariaco

Lagerstroemia indica L. (Lythraceae) - astronomía

Lagerstroemia speciosa (L.) Persoon (Lythraceae) - reina de las flores

Lansium domesticum Corrêa da Serra (Meliaceae) - lanzón

Laurus nobilis L. (Lauraceae) - laurel

Leonotis leonurus (L.) R. Brown (Lamiaceae) oreja-de-león

Litchi chinensis Sonnerat (Sapindaceae) - lychee

*Lysiloma latisiliqua (L.) Bentham (Fabaceae) tamarindo silvestre

Lysiloma sabicu Bentham (Fabaceae) - quebracho

Macadamia integrifolia Maiden \& Betche

(Proteaceae) - nuez de macadamia

Macadamia tetraphylla L. A. S. Johnson

(Proteaceae) - nuez de macadamia

Mangifera indica L. (Anacardiaceae) - mango

Manilkara jaimiqui (C. Wright) Dubard

(Sapotaceae) - jaimiquí

Manilkara zapota (L.) van Royen (Sapotaceae) zapote chico, níspero

*Mastichodendron foetidissimum (Jacquin) H. J.

Lam (Sapotaceae) - jocuma, tortugo amarillo

Melaleuca quinquenervia (Cavanilles) S.T. Blake (Myrtaceae) - cajeput

Melaleuca decora (Salisbury) Britten (Myrtaceae) - árbol de té

Melicocca bijuga L. (Sapindaceae) - mamoncillo

Michelia champaca L. (Magnoliaceae) - magnolia tropical

Mimusops elengi L. (Sapotaceae) - balatá, ausubo 
Mussaenda erythrophylla Schumacher \& Thonning (Rubiaceae) - Flor de trapo

Myrciaria cauliflora (de Candolle) Berg in Martius - jaboticaba

*Myrica cerifera L. (Myricaceae) - palo de cera

*Myrsine guianensis (Aublet) Kuntze

(Myrsinaceae) - bádula

*Nectandra coriacea (Swartz) Grisebach

(Lauraceae) - laurel avispillo

Ocimum sp. (Lamiaceae) - albahaca tailandesa

Pachystachys lutea Nees (Acanthaceae) -

langostinos dorados

*Parthenocissus quinquefolia (L.) Planchon - viña de Virginia

Peltophorum pterocarpum (De Candolle) K. Heyne (Fabaceae) - vaina de cobre

Persea americana Miller (Lauraceae) - aguacate

*Persea borbonia (L.) Sprengel (Lauraceae) laurel rojo

Petrea volubilis L. (Verbenaceae) - pétrea

Phoenix roebelenii O'Brien (Palmae) - palma datilera miniatura

Pimenta dioica (L.) Merrill (Myrtaceae) pimentón

Pimenta racemosa (Miller) J. Moore (Myrtaceae) malagueta

Pithecellobium flexicaule (Bentham) J.M. Coulter (Fabaceae) - ébano tejano

Polygala cowellii (Britton) Blake (Polygalaceae) palo de violeta

Pongamia pinnata (L.) Pierre (Fabaceae) - pongam

Psidium littorale Raddi (Myrtaceae) - araza

* Psychotria ligustrifolia (Northrop) Millspaugh café silvestre enano (Rubiaceae)
* Psychotria nervosa Swartz (Rubiaceae) - café silvestre

*Quercus geminata Small (Fagaceae) - encino del arena

*Quercus lauirifolia Michaux (Fagaceae) encino-laurel

Quercus virginiana Miller (Fagaceae) - encino de Virginia

Rheedia aristata Grisebach (Clusiaceae) - manajú

Rheedia sp.

Rhizophora mangle (L.) - mangle rojo

Ruellia sp. (Acanthaceae)

Rondeletia leucophylla Kunth (Rubiaceae)

Rosa sp. (Roseaceae) - rosa

*Salix caroliniana Michaux (Salicaceae) - sauce de los llanos de la costa

Samanea saman (Jaquin) Merrill (Fabaceae) samán

Schinus terebinthifolius Raddi (Anacardiaceae) pimienta brasilera

Synsepalum dulcificum Schumacher \& Thonning (Sapotaceae) - fruta milagrosa

Syzigium cuminii (L.) Skeels (Myrtaceae) jambolán

Syzigium paniculatum Gaertner (Myrtaceae)

Terminalia muelleri Bentham (Combretaceae)

Terminalia catappa L. (Combretaceae) - almendra tropical

*Trema micrantha (L.) Blume (Celtidaceae) guacimilla 


\section{Cuadro 2. Lista de plantas hospedantes de escama lobada de laca hasta Octubre, 2002}

Lista compilada por Bill Howard, Dave McLean, Bob Pemberton, Avas Hamon, Greg Hodges, y Jeanette Wofford. Contribuidores adicionales: John Cannon, Chris y Karolynne Griffiths, Way Hoyt, Neal Kolnik, Andrew Southerland y Laura Tooley.

* indica especies nativas á la Florida.

\section{Acanthaceae}

Pachystachys lutea Nees - langostinos dorados

Ruellia sp.

Aceraceae

*Acer rumbrum L. - arce rojo

Aquifoliaceae

Ilex vomitoria Aiton - acebo yaupon

\section{Anacardiaceae}

Mangifera indica L. - mango

Schinus terebinthifolius Raddi - pimienta brasilera

\section{Annonaceae}

Annona cherimola Miller X A. squamosa L. atemoya

*Annona glabra L. - anón de agua

Annona muricata L. - guanábana

Annona reticulata L. - mamón

Annona squamosa L. - anón

Cananga odorata (Lamarck) J. D. Hooker \& T.

Thomson (Annonaceae) - ilan-ilan

\section{Asteraceae}

*Baccharis halimifolia L. - saltbush, carqueja

\section{Burseraceae}

*Bursera simaruba (L.) Sargent - almácigo

Capparaceae

*Capparis cynophallophora L. - burro prieto

\section{Casuarinaceae}

Casuarina equisetifolia L. - casuarina

\section{Celtidaceae}

Celtis laevigata Willdenow - palo blanco

Trema micrantha (L.) Blume - guacimilla

Chrysobalanaceae

*Chrysobalanus icaco L. - icaco

\section{Clusiaceae}

Calophyllum brasiliense Cambessédes - palo maría

*Clusia rosea Jacquin - cupey

Garcinia prainiana King - mangostán botón

Rheedia aristata Grisebach - manajú

Rheedia sp.

\section{Combretaceae}

Bucida buceras L. - ucar

*Conocarpus erectus L. - mangle botón

Terminalia muelleri Bentham (Combretaceae)

Terminalia catappa L. (Combretaceae) - almendra tropical

\section{Cupressaceae}

Juniperus silicicola (Small) Bailey - enebro rojo del sur

\section{Ebenaceae}

Diospyros digyna Jacquin - zapote negro

Elaeocarpaceae 
Elaeocarpus sp.

\section{Euphorbiaceae}

Acalypha wilkesiana J. Mueller

(Eurphorbiaceae) - acalifa

Antidesma bunius (L.) Sprengel - bignay

\section{Fabaceae}

Acacia auriculiformis Bentham - acacia de hoja-oreja

Bauhinia sp. - árbol de las orquídeas

Brya ebenus (L.) De Candolle - granadillo

Calliandra haematocephala Hasskarl - caliandra

Calliandra surinamensis Bentham - caliandra rosa

Inga affinis de Candolle - ingá dulce

Igna edulis Martius - guamo *Lysiloma latisiliqu

(L.) Bentham - tamarindo silvestre

Lysiloma sabicu Bentham - quebracho

Peltophorum pterocarpum (De Candolle) K.

Heyne - vaina de cobre

Pithecellobium flexicaule (Bentham) J.M. Coulter ébano tejano

Pongamia pinnata (L.) Pierre - Pongam

Fagaceae

*Quercus geminata Small - encino de arena

*Quercus laurifolia Michaux (Fagaceae) - encino laurel

*Quercus virginiana Miller (Fagaceae) - encino de Virginia

\section{Flacourtiaceae}

Dovyalis hebecarpa (G. Gardner) Warburg grosella de Ceilán

\section{Lamiaceae}

Leonotis leonurus (L.) R. Brown - oreja de león

Ocimum sp. - albahaca tailandesa

\section{Lauraceae}

Cinnamomum zeylanicum Blume - canela

Laurus nobilis L. - laurel

Nectandria coriacea (Swartz) Grisebach - laurel avispillo

Persea americana Miller - aguacate

*Persea borbonia (L.) Sprengel - laurel rojo

\section{Lythraceae}

Lagerstroemia indica L. - astronomía, crespón, árbol de Júpiter

Lagerstroemia speciosa (L.) Persoon (Lythraceae)

- reina de las flores

\section{Magnoliaceae}

Michelia champaca L. - magnolia tropical

Malvaceae

Hibiscus rosa-sinensis L. - hibisco

Meliaceae

Lansium domesticum Corrêa da Serra - lanzón

\section{Moraceae}

Brosimum alicastrum Swartz - ramón, cacique

*Ficus aurea Nuttall - jagüey estrangulador

Ficus benjamina L. - caucho

Ficus microcarpa L.f. - laurel de la India

Ficus nota (Blanco) Merrill - tibig

Ficus rubiginosa Ventenat - jagüey herrumbroso

Ficus salicifolia (Vahl) Berg - jagüey de hoja de sauce 


\section{Myrsinaceae}

*Ardisia escallanoides Schlechtendal \& Chamisso baya de marga

*Myrsine guianensis (Aublet) Kuntze - bádula

\section{Myricaceae}

*Myrica cerifera L. - palo de cera

\section{Myrtaceae}

Callistemon viminalis (Gaertner) Cheel - calistemon

*Calyptranthes pallens Grisebach - limoncillo

*Calyptranthes zuzygium (L.) Swartz - arrayán del rió

*Eugenia axillaris (Swartz) Willdenow - guairaje

Eugenia brasiliensis Lamarck - grumichama

Eugenia confusa de Candolle - arrayán de baya roja

Eugenia uniflora L. - pitanga

Melaleuca quinquenervia (Cavanilles) S.T. Blake cajeput

Melaleuca decora (Salisbury) Britten - árbol de té

Myrciaria cauliflora (de Candolle) Berg in Martius

- jaboticaba

Pimenta dioica (L.) Merrill - pimentón

Pimenta racemosa (Miller) J. Moore - malagueta

Psidium littorale Raddi - araza

Syzigium cuminii (L.) Skeels - jambolan

Syzigium paniculatum Gaertner

\section{Oxalidaceae}

Averrhoa carambola L. - carambola

\section{Palmae}

Phoenix roebelenii O'Brien - palma datilera miniatura

\section{Polygalaceae}

Polygala cowellii (Britton) Blake - árbol de violeta

\section{Proteaceae}

Macadamia integrifolia Maiden \& Betche - nuez de macadamia

Macadamia tetraphylla L. A. S. Johnson - nuez de macadamia

\section{Rhamnaceae}

*Krugiodendron ferreum (Vahl) Urban - bariaco

\section{Rhizophoraceae}

Rhizophora mangle (L.) - mangle rojo

\section{Roseaceae}

Rosa sp. - rosa

\section{Rubiaceae}

Gardenia jasminoides Ellis - gardenia

Hamelia cuprea Grisebach - Bahamas flame bush

*Hamelia patens Jacquin - coloradillo

Mussaenda erythrophylla Schumacher \& Thonning

- flor de trapo

*Psychotria ligustrifolia (Northrop) Millspaugh café silvestre enano

*Psychotria nervosa Swartz - café silvestre

Rondeletia leucophylla Kunth

\section{Rutaceae}

Citrus X paradisi Macfadyen - toronjo

Clausena lansium (Loureiro) Skeels - wampi

Fortunella japonica (Thunberg) Swingle - naranjito

\section{Salicaceae}

* Salix caroliniana Michaux - sauce de los llanos de la costa 


\section{Sapindacea}

Blighia sapida Konig - acki

Cupaniopsis anacardioides (A. Richard) Radlkofer

- carrotwood

*Exothea paniculata (Jussieu) Radlkofer -

guamaca, yaicuaje

Filicium decipiens (Wight \& Arnot) Thwaites árbol-helecho japonés

Litchi chinensis Sonnerat - lychee

Melicocca bijuga L. - mamoncillo

\section{Sapotaceae}

Bumelia celastrina Humboldt, Bonpland \& Kunth coma

Chrysophyllum cainito L. - caimito

*Chrysophyllum oliviforme L. - caimitillo

*Dipholis salicifolia (L.) Alphonse de Candolle zitzia

Manilkara zapota (L.) van Royen - níspero

Manilkara jaimiqui (C. Wright) Dubard - jaimiquí

*Mastichodendron foetidissimum (Jacquin) H. J.

Lam ) - jocuma, tortugo amarillo

Mimusops elengi L. - balatá, ausubo

Synsepalum dulcificum Schumacher \& Thonning ) fruta milagrosa

\section{Solanaceae}

Brunfelsia sp.

Brunfelsia nitida Bentham - dama de noche

Cestrum nocturnum L. - galán de noche

\section{Tiliaceae}

Grewia occidentalis L. - flor-estrella

Ulmaceae
*Trema micranthum (L.) Blume - guacimilla

Verbenaceae

Avicennia germinans (L.) L. - mangle negro

Petrea volubilis L. - pétrea

Vitaceae

*Parthenocissus quinquefolia (L.) Planchon - viña de Virginia

\section{Expansión potencial en la distribución}

El potencial de que este insecto se disperse y ocupe aún más territorio en el hemisferio occidental es especialmente alto para áreas cálidas y donde hay importación a un nivel significante de plantas vivas desde Florida, e. g., Puerto Rico y otras localidades del Caribe, California, y Hawaii. La invasión de áreas naturales es una preocupación primordial. Un examen rápido de varios montecillos de árboles de madera dura en el Condado de Broward reveló que habían infestaciones densas en diversas especies en áreas grandes. La presencia de infestaciones densas $28 \mathrm{~km}$ al interior, eso es, casi en las orillas de los Everglades, implica que la vegetación de esta área natural vasta está amenazada. La mayoría de las plantas hospedantes nativas identificadas en Florida también están distribuidas en la Región Caribeña, y si el insecto fuese introducido a Puerto Rico u otros países caribeños, las áreas naturales de esta región también serían amenazadas.

\section{Prospectos para el manejo de la plaga}

No se ha publicado virtualmente nada sobre la biología y el manejo de esta especie. Esta ha llegado rápidamente a ser una plaga de mayor importancia en el sureste de la Florida, y es urgente el acelerar las investigaciones sobre ella. Raramente hemos encontrado insectos parasíticos que ataquen a $P$. lobata, y concluimos que estos no ofrecen control biológico significante. Resultados no publicados de experimentos llevados a cabo por la Universidad de Florida, Centro de Investigaciones y Enseñanza de 
Fort Lauderdale, mostraron que un remojo de las raíces con el insecticida imidacloprid controló efectivamente a $P$. lobata infestando árboles grandes de caucho (Ficus benjamina). Estudios adicionales se llevan a cabo para refinar este método, y para hacer pruebas de la efectividad de aceites parafínicos además de otros métodos químicos. Como se pronostica que un alto porcentaje de árboles y arbustos llegarán a estar infestados con este insecto en áreas urbanas al igual que en áreas naturales de la Florida, estamos investigando el control biológico como la única opción a largo plazo.

\section{Referencias seleccionadas}

Hamon A. (14 June 2001). Lobate lac scale, Paratachardina lobata lobata (Chamberlin) (Hemiptera: Kerriidae). Pest Alert. http://www.doacs.state.fl.us/ pi/enpp/ento/ paratachardina.html (30 October 2002).

Miller DR, Ben-Dov Y. (16 August 2002) ScaleNet http://www.sel.barc.usda.gov/scalenet/ scalenet.htm (30 October 2002). 